\title{
Correlação entre métodos tradicionais e espectroscopia de ultra-som na determinação de características físico-químicas do leite
}

[Correlation between traditional methods and ultrasonic spectroscopy for measurement of milk physical-chemical characteristics]

\author{
E.H.G. Ponsano ${ }^{1}$, S.H.V. Perri ${ }^{1}$, F.C.P. Madureira ${ }^{2}$, R.Z. Paulino ${ }^{1}$, L.G. Camossi ${ }^{1}$ \\ ${ }^{1}$ Departamento de Apoio, Produção e Saúde Animal - UNESP \\ Rua Clóvis Pestana, 793 \\ 16050-680 - Araçatuba, SP \\ ${ }^{2}$ Cooperativa de Laticínios Campezina - Penápolis, SP
}

\begin{abstract}
RESUMO
Compararam-se a espectroscopia de ultra-som (US) e os métodos tradicionais (MT) utilizados para a determinação de características físico-químicas do leite e estimaram-se as correlações entre esses métodos e suas acurácias. As características densidade, extrato seco desengordurado (ESD), teor de proteínas e teor de gordura foram determinadas em 65 amostras de leite cru por ambos os métodos. As densidades médias determinadas pelo US e pelos MT não diferiram entre si $(\mathrm{P}=0,14)$, e a correlação encontrada entre os dois métodos para a determinação da densidade não foi significativa $(\mathrm{P}=0,08)$. Os teores médios de ESD, proteína e gordura encontrados pelo US e pelos MT foram diferentes $(\mathrm{P}=0,04, \mathrm{P}<0,0001$ e $\mathrm{P}<0,0001$, respectivamente), as correlações entre os dois métodos utilizados para a análise dessas características foram positivas e significativas $(\mathrm{r}=0,0109, \mathrm{r}=0,0007, \mathrm{r}=<0,0001$, respectivamente) e as acurácias dos métodos para essas determinações foram de $0,160,0,062$ e 0,145 , respectivamente. Foi determinada a equação de regressão linear, que associa o teor de gordura obtido no método de espectroscopia de ultra-som ao do método butirométrico, que apresentou coeficiente de determinação de 0,5936 .
\end{abstract}

Palavras-chave: leite, análise físico-química, ultra-som, método tradicional, correlação

\begin{abstract}
The ultrasonic spectroscopy (US) and the traditional methods (TM) used for the measurement of milk physical-chemical characteristics were studied and the correlations between these methodologies and their accuracies were estimated. Milk density, solids-non-fat (SNF), protein and fat contents were determined in 65 raw milk samples by both methods. Means of density determined by US and TM did not differ $(P=0.14)$, although no significant correlation was found $(P=0.08)$ between the methods used for the determination of this characteristic. Means of SNF, protein and fat contents determined by US and TM differed ( $P=0.04, P<0.0001$ and $P<0.0001$, respectively). Correlations between both methods used for the analysis of these characteristics were positive and significant $(r=0.0109, r=0.0007$ and $r=<0.0001$, respectively), and the accuracies of the methods for these analysis were 0.160, 0.062 and 0.145, respectively. The regression equation of US results on TM for fat content showed a coefficient of determination of 0.5936 .
\end{abstract}

Keywords: milk, physical-chemical analysis, ultrasound, traditional method, correlation

Recebido em 26 de janeiro de 2006

Aceito em 14 de abril de 2007

E-mail: elisahgp@fmva.unesp.br 


\section{INTRODUÇÃO}

A Instrução Normativa $\mathrm{n}^{\circ} 51$ (NI 51) do Ministério da Agricultura, Pecuária e Abastecimento - MAPA (Brasil, 2002) estabelece os novos padrões de identidade e qualidade para os diferentes tipos de leite comercializados no Brasil. De acordo com a IN 51, todo leite cru produzido no Brasil deverá ter sua qualidade monitorada, sendo a freqüência das análises, as características a serem avaliadas e os padrões normais estipulados para cada tipo de leite. Esse monitoramento já vem sendo realizado por laboratórios oficiais credenciados junto ao MAPA, os quais se encontram equipados para a determinação das características de qualidade exigidas pela legislação. Paralelamente, os estabelecimentos beneficiadores de leite também realizam o controle de qualidade do leite de seus fornecedores como forma de avaliar o atendimento aos padrões de composição e higiene preconizados pela legislação $\mathrm{e}$, conseqüentemente, assegurar ao consumidor um produto de qualidade, sob os aspectos físicoquímicos e microbiológicos. Também, por meio de ações de controle de qualidade da matériaprima, torna-se possível ao laticínio estabelecer um sistema de pagamento diferenciado ao produtor como forma de incentivo à produção de leite de qualidade. No entanto, para atender a essa legislação, o número de amostras a serem analisadas nos estabelecimentos beneficiadores bem como a freqüência da realização das análises tendem a aumentar, tornando imperativa a utilização de métodos analíticos que expressem resultados seguros em curto espaço de tempo.

Em países desenvolvidos, a remuneração ao produtor baseia-se na qualidade do leite. Desse modo, houve a necessidade de aperfeiçoar os métodos de análise, o que produziu uma investigação dirigida à criação de procedimentos analíticos automatizados, mais rápidos $\mathrm{e}$ adequados para o trabalho em série (Buckin et al., 2003). Os equipamentos desenvolvidos para essa finalidade vêm, agora, atender à nova demanda verificada no Brasil.

A tecnologia ultra-sônica foi desenvolvida a partir do princípio fundamental de que a matéria é capaz de absorver o som, atenuando-o ou alterando sua velocidade. A partir daí, a espectroscopia ultra-sônica expandiu sua faixa de aplicação, até chegar à caracterização de misturas físicas e químicas (Kinsler et al., 1982; O'Driscoll et al., 2003). De acordo com Gunasekaran e Ay (1994), as primeiras referências a respeito do uso de ultra-som na indústria de alimentos datam de 1961, com a aplicação do método para a medida de sólidos não gordurosos e gordura do leite.

O uso de técnicas espectroscópicas para a análise dos componentes do leite é uma ferramenta importante por fornecer, em tempo reduzido, informações úteis para os produtores de leite, que podem utilizá-las para detectar problemas de manejo nutricional e, assim, aumentar a eficiência produtiva dos rebanhos (Tsenkova et al., 2000). O uso dessas técnicas é, também, de fundamental importância para os estabelecimentos beneficiadores de leite, que podem definir o destino a ser dado ao leite, subsidiar o pagamento diferenciado ao produtor com base na qualidade e assegurar que seu produto esteja adequado às normas de composição de leite exigidas pela legislação.

A espectroscopia de ultra-som baseia-se no princípio físico de que o movimento de qualquer onda é afetado pelo meio por onde ela está se propagando (Nelligan, 2003). Dessa forma, a propagação das ondas sonoras em um determinado meio fornece informações sobre ele pela análise da transmissão ou da reflexão dos sinais gerados (Bhardwaj, 2002). Essa técnica emprega ondas sonoras de alta freqüência que imprimem forças intermoleculares aos materiais em teste. As oscilações de compressão ou descompressão das ondas ultra-sônicas causam oscilações no arranjo molecular da amostra, que responde com forças de atração ou repulsão intermoleculares (Buckin et al., 2003). As amplitudes de deformação nas ondas ultrasônicas empregadas na determinação são extremamente pequenas, tornando a técnica não destrutiva, o que representa uma oportunidade única na caracterização de produtos alimentícios de base líquida, incluindo amostras opacas como o leite (Buckin et al., 2003; Dukhin et al., 2003; Nelligan, 2003).

A IN 51 (Brasil, 2002) estabelece as características físico-químicas a serem avaliadas no leite cru destinado ao processamento, bem como os métodos de referência para a análise de cada um deles. A IN 51 estabelece, também, que 
tais métodos poderão ser substituídos por outros métodos de controle operacional, desde que sejam conhecidos seus desvios e correlações em relação aos respectivos métodos de referência.

A literatura nacional carece de informações a respeito da correlação entre os métodos tradicionalmente utilizados na determinação de componentes físico-químicos do leite e a espectroscopia acústica.

Este trabalho teve como objetivos comparar as metodologias tradicionais e a espectroscopia de ultra-som para a avaliação das características do leite (densidade, gordura, proteínas e extrato seco desengordurado) e estimar a correlações entre elas e suas acurácias.

\section{MATERIAL E MÉTODOS}

Entre outubro de 2004 e junho de 2005, coletaram-se 65 amostras de leite cru, provenientes de propriedades integrantes da Cooperativa de Laticínios Campezina (Penápolis, SP). Depois de adequada agitação, as amostras foram coletadas diretamente dos tanques de expansão localizados nas propriedades produtoras e transportadas em recipientes isotérmicos até o laboratório de controle de qualidade do laticínio, onde foram realizadas as determinações de densidade a $15^{\circ} \mathrm{C}$, gordura, proteínas e extrato seco desengordurado (ESD), em analisador ultra-sônico ${ }^{1}$ (US), calibrado para a análise de leite de vaca. As determinações foram realizadas em duplicata, em temperatura ambiente, utilizando-se $5 \mathrm{ml}$ de cada uma das amostras, em temperatura entre 15 e $20^{\circ} \mathrm{C}$.

Após esse procedimento, as amostras foram imediatamente transportadas, em recipientes isotérmicos, ao Laboratório de Análise de Alimentos do Curso de Medicina Veterinária da Unesp - Araçatuba, SP -, onde foram analisadas por meio de técnicas analíticas tradicionais (MT): a densidade, a $15^{\circ} \mathrm{C}$, foi determinada utilizando-se termolactodensímetro de Quennève, com as amostras entre 10 e $20^{\circ} \mathrm{C}$ (Laboratório..., 1981); a porcentagem de gordura foi determinada pelo método butirométrico de Gerber (International..., 1991); o teor de proteínas foi determinado por meio da técnica de
micro-Kjeldahl (International..., 1993), multiplicando-se o teor de nitrogênio encontrado pelo fator de conversão 6,38; o ESD foi calculado a partir da subtração da porcentagem de gordura do valor de extrato seco total (EST); o EST foi determinado indiretamente com o auxílio de disco de Ackermann, a partir dos valores obtidos para porcentagem de gordura $\mathrm{e}$ densidade a $15^{\circ} \mathrm{C}$ (Laboratório..., 1981).

A comparação entre os valores de densidade, ESD, proteínas e gordura foi realizada por meio do teste t para amostras pareadas, correlação e regressão linear (Zar, 1984), utilizando-se o programa SAS (Statistical..., 1992). O nível de significância adotado foi de 5\%. A acurácia dos métodos foi estimada pelo erro-padrão residual $(\mathrm{s}(\mathrm{y}, \mathrm{x}))$, conforme recomendado por IDF (International...,1999).

\section{RESULTADOS E DISCUSSÃO}

Não houve diferença significativa $(\mathrm{P}=0,4)$ entre as densidades médias obtidas pelo US e pelo MT (Tab.1). A correlação entre os métodos avaliados não foi significativa $(\mathrm{P}=0,0804)$, e o coeficiente de correlação encontrado entre eles foi de 0,225. A determinação dessa variável pelo método tradicional é passível de erros, pois depende da interpretação visual do analista, da calibração do termolactodensímetro e da correção do valor encontrado para a temperatura padrão de $15^{\circ} \mathrm{C}$. Esses fatores podem ter contribuído para a nãosignificância da correlação entre US e MT. A espectroscopia ultra-sônica baseia-se na avaliação do comportamento de determinados parâmetros das ondas sonoras de alta freqüência ao se propagarem através de uma amostra (Bhardwaj, 2002; Dukhin et al., 2003). A velocidade do som é, geralmente, o parâmetro ultra-sônico mais facilmente medido e, por isso, empregado na maioria dos equipamentos ultrasônicos (Nelligan, 2003). No caso da espectroscopia ultra-sônica, a densidade do leite está diretamente relacionada à medida da velocidade ultra-sônica, parâmetro extremamente sensível à organização molecular e às interações intermoleculares da amostra, fornecendo, assim, informações bastante seguras sobre a concentração dos componentes (Buckin et al., 2003; Nelligan, 2003).

${ }^{1}$ Ekomilk-Milkana Stara - Zagora, Bulgária 
Tabela 1. Médias ( $\bar{x}$ ), desvios-padrão (s) e coeficientes de correlação (r) da densidade, extrato seco desengordurado (ESD), proteína e gordura, analisadas por espectroscopia ultra-sônica (US) e pelos métodos tradicionais (MT), e níveis descritivos dos testes t pareados $\left(\mathrm{P}^{1}\right)$ e das correlações lineares $\left(\mathrm{P}^{2}\right)$

\begin{tabular}{lccccc}
\hline Variável & US $(\bar{x} \pm \mathrm{s})$ & $\mathrm{MT}(\bar{x} \pm \mathrm{s})$ & $\mathrm{P}^{1}$ & $\mathrm{R}$ & $\mathrm{P}^{2}$ \\
\hline Densidade & $1,032 \pm 0,001$ & $1,032 \pm 0,001$ & 0,1398 & 0,225 & 0,0804 \\
ESD & $9,017 \pm 0,168$ & $8,965 \pm 0,172$ & 0,0448 & 0,324 & 0,0109 \\
Proteína & $3,241 \pm 0,069$ & $3,096 \pm 0,207$ & $<0,0001$ & 0,444 & 0,0007 \\
Gordura & $3,437 \pm 0,225$ & $3,547 \pm 0,303$ & $<0,0001$ & 0,770 & $<0,0001$ \\
\hline
\end{tabular}

A determinação do extrato seco desengordurado do leite fornece informações a respeito da prática de aguagem (Fagundes, 1997). Os valores de ESD médios encontrados foram diferentes $(\mathrm{P}=0,04)$, porém a correlação entre os métodos avaliados foi significativa $(\mathrm{P}<0,05)$, com coeficiente de correlação de 0,324 (Tab. 1) e acurácia de 0,160 . Convém lembrar que, para a determinação do ESD pelo MT, é necessária a determinação prévia do EST que, por sua vez, depende dos valores de densidade e porcentagem de gordura. Portanto, qualquer erro na determinação dessas variáveis pelos MT levará, diretamente, a erros nos valores de ESD, o que pode justificar as diferenças estatísticas nas médias dos resultados encontrados para os dois métodos.

O conhecimento do teor de proteínas no leite fornece informações sobre parâmetros nutricionais e metabólicos, além de constituir um dado fundamental para a produção de derivados (Amiot, 1991; Spreer, 1991; Tsenkova et al., 2000; Fonseca e Santos, 2001). Os teores médios de proteínas diferiram $(\mathrm{P}<0,001)$ entre os dois métodos de análise estudados, provavelmente por influência da avaliação subjetiva realizada durante a etapa de titulação na técnica de determinação de proteínas pelo MT, quando o ponto final da reação se baseia na visualização da mudança de cor do indicador pelo analista. Esse mesmo fator pode justificar, também, o maior desvio-padrão encontrado para o MT na determinação de proteínas, em comparação com o US. A correlação entre os métodos avaliados foi significativa $(\mathrm{P}=0,1)$, com coeficiente de correlação de 0,444 (Tab. 1) e acurácia de 0,062. $\mathrm{O}$ método de Kjeldahl mede a concentração de nitrogênio orgânico total da amostra, sendo, posteriormente, convertido para concentração de proteínas. Isso significa que o nitrogênio proveniente de fontes não-protéicas, tais como ácidos nucléicos, alcalóides, porfirinas, pigmentos, lipídeos e carboidratos nitrogenados, também entra no cômputo de proteína total (Cecchi, 2003). Porém, no caso do leite, esses compostos nitrogenados não-protéicos estão ausentes ou presentes em concentrações insignificantes, o que torna o método bastante apropriado para a estimativa do conteúdo protéico. Tem-se como inconveniente, entretanto, o fato de ser uma técnica laboriosa e demorada, além de requerer manipulação especializada, equipamentos e vidrarias próprios, geralmente onerosos, e de consumir ácidos e base, gerando resíduos químicos laboratoriais em grande quantidade. $\mathrm{Na}$ espectroscopia de ultrasom, a concentração de colóides é determinada a partir da avaliação dos distúrbios mecânicos provocados pelas ondas sonoras ao passar pela amostra, sendo recomendada por pesquisadores da área para a análise de proteínas em leite e derivados (Miles et al. 1990; Buckin et al., 2003).

A porcentagem de gordura presente no leite pode fornecer informações a respeito da prática de desnatamento, além de permitir a avaliação nutricional da dieta fornecida ao rebanho, o metabolismo dos animais na conversão da dieta e a avaliação do manejo da ordenha. (Amiot, 1991; Spreer, 1991; Tsenkova et al., 2000; Fonseca e Santos, 2001). Além disso, essa informação também serve como base para pagamento aos produtores em alguns estabelecimentos beneficiadores de leite. Pelos dados apresentados na Tab.1, verifica-se que a correlação entre US e MT para a porcentagem de gordura $(r=0,770)$ foi elevada $(\mathrm{P}<0,001)$, com alto coeficiente de correlação e acurácia de 0,145. As médias encontradas para essa variável diferiram $(\mathrm{P}<0,001)$ entre os dois métodos estudados. A determinação da gordura em leite pelo MT envolve uma reação de hidrólise ácida para separar proteínas e carboidratos ligados aos glóbulos de gordura (Cecchi, 2003). A técnica analítica do MT utiliza ácido sulfúrico e álcool amílico, gerando um resíduo químico que requer 
descarte apropriado, nem sempre possível de ser realizado nos laboratórios de controle de qualidade. É também técnica destrutiva e requer equipamento e vidrarias específicos. Outro parâmetro medido na espectroscopia de ultrasom, a atenuação, é determinado pela dispersão de ondas ultra-sônicas em amostras nãohomogêneas, tais como emulsões e suspensões, e está diretamente envolvido na análise da concentração da gordura do leite por esse método (Bhardwaj, 2002; Buckin, et al., 2003; Dukhin et al., 2003). Miles et al. (1990) pesquisaram a atenuação provocada pela fração gordurosa em leite integral submetido a diferentes pressões durante a operação de homogeneização e em leite desnatado e verificaram que a atenuação do ultra-som depende não somente da concentração de lipídeos, como também do grau de homogeneização, em razão de perdas térmicas resultantes da transferência de calor do meio aquoso para os glóbulos de gordura e vice-versa.

Na Fig. 1 são apresentados a dispersão, a equação de regressão e o respectivo coeficiente de determinação para porcentagem de gordura, obtidos pela espectroscopia de ultra-som e pelo método tradicional. $\mathrm{O}$ coeficiente de determinação encontrado para essa variável indica que $59,4 \%$ da variabilidade do US é explicada pelo MT.

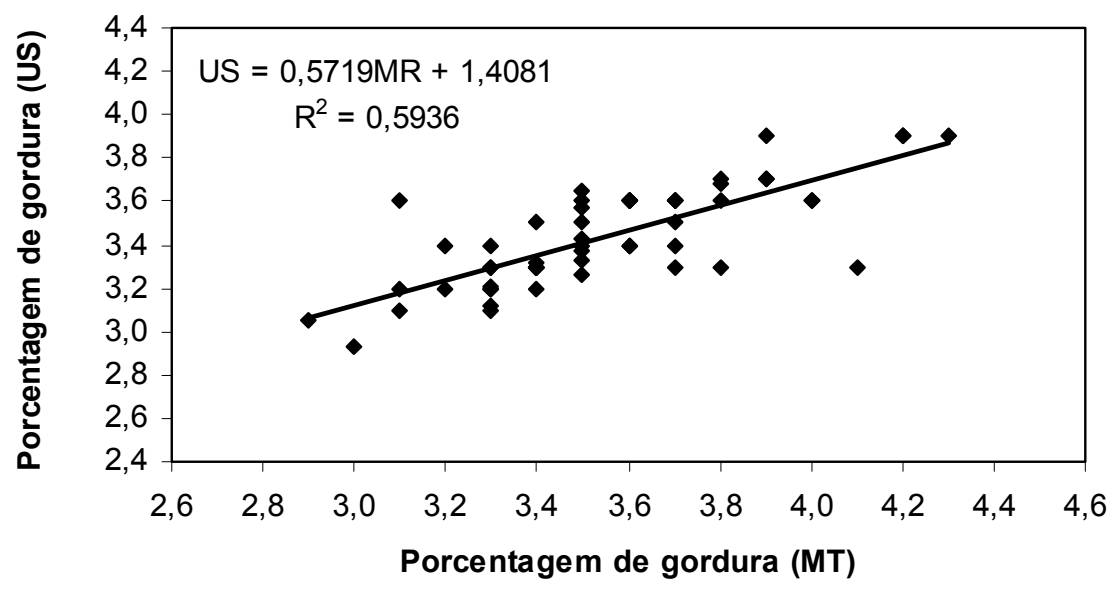

Figura 1. Diagrama de dispersão da porcentagem de gordura entre a espectroscopia de ultra-som (US) e a metodologia tradicional (MT) com representação da reta de regressão e coeficiente de determinação.

Embora uma discussão mais aprofundada sobre o assunto esteja inviabilizada em razão da falta de estudos na literatura a respeito da comparação entre a espectroscopia de ultra-som e os métodos tradicionais na determinação de características físico-químicas do leite, diversos autores relatam que a análise ultra-sônica em leite e derivados é bastante útil em fornecer informações sobre a composição e a estrutura dos componentes físico-químicos, importantes para o controle do processamento e da qualidade (Miles et al., 1990; Buckin et al., 2003; Dukhin et al., 2003; Nelligan, 2003).

Convém salientar que, em termos operacionais, a análise físico-química do leite por espectroscopia de ultra-som apresenta vantagens sobre os métodos tradicionais por dispensar o preparo das amostras, utilizar volumes mínimos das amostras em estado intacto, dispensar o uso de reagentes químicos e vidrarias específicos e por fornecer o resultado em poucos minutos. Os resultados apresentados fornecem subsídios para a tomada de decisões por parte de estabelecimentos beneficiadores e órgãos regulamentadores e fiscalizadores de leite e derivados no direcionamento da escolha dos métodos de controle de qualidade a serem praticados.

\section{AGRADECIMENTOS}

À Reitoria da UNESP, pelas bolsas de Iniciação Científica. 


\section{REFERÊNCIAS BIBLIOGRÁFICAS}

AMIOT, J. Ciencia y tecnologia de la leche. Zaragoza: Acribia, 1991. 547p.

BHARDWAJ, M.C. Non contact ultrasound: the final frontier in non destructive analysis. Boalsburg: Second Wave Systems, 2002. 50p.

BRASIL. Instrução Normativa $n^{\circ} 51$ de 18 set. 2002. Regulamentos técnicos de produção, identidade, qualidade, coleta e transporte de leite. Diário Oficial da União, Brasília, n.183, 20 set. 2002, seção 1, p.13-22.

BUCKIN, V.; O’DRISCOLL, B.; SMYTH, C. Ultrasonic spectroscopy for material analysis: recent advances. Spectrosc. Eur., v.15, p.20-25, 2003.

CECCHI, H. M. Fundamentos teóricos e práticos em análise de alimentos. 2.ed. Campinas: Unicamp, 2003. 207p.

DUKHIN, A.S.; GOETZ, P.J.; TRAVERS, B. Ultrasound for characterizing liquid based food products. 1 - acoustic spectroscopy. Mount Kisco: Dispersion Technology, 2003. 26f.

FAGUNDES, C.M. Inibidores e controle de qualidade do leite. 2.ed. Pelotas: Universitária, 1997. 115p.

FONSECA, L.F.L.; SANTOS, M.V. Qualidade do leite e controle de mastite. 2.ed. São Paulo: Lemos, 2001. 175p.

GUNASEKARAN, S.; AY, C. Evaluating milk coagulation with ultrasonics. Chicago, 1993. Disponível em: http://144.92.76.98/Guna/evaluatingmilk94.>. Acessado em: 21 set. 2006.

INTERNATIONAL Dairy Federation. Fat content in milk - butyrometric method. Standard 152. Bruxelas: IDF, 1991.

INTERNATIONAL Dairy Federation. Milk. Determination and evaluation of the overall accuracy of indirect methods of milk analysis application to calibration procedure and quality control in the dairy laboratory. Standard 128A. Bruxelas: IDF, 1999.

INTERNATIONAL Dairy Federation. Milk. Determination of the total nitrogen content. Standard 20B. Bruxelas: IDF, 1993.

KINSLER, L.E.; FREY, A.R.; COPENS, A.B. et al. Fundamentals of Acoustics. 3.ed. New York: John Wiley \& Sons, 1982.

LABORATÓRIO Nacional de Referência Animal. Métodos analíticos oficiais para controle de produtos de origem animal e seus ingredientes. Brasília: Ministério da Agricultura, 1981. v.2.

MILES, C.A.; SHORE, D.; LANGLEY, K.R. Attenuation of ultrasound in milks and creams. Ultrasonics, v.28, p.394-400, 1990.

NELLIGAN, T.J. An introduction to ultrasonic material analysis. Waltham: General Electric, 2003. 3f.

O’DRISCOLL, B.; SMYTH, C.; ALTING, A.C. et al. Recent applications for high-resolution ultrasonic spectroscopy. Am. Lab., p.54-57, 2003.

SPREER, E. Lactologia industrial. 2.ed. Zaragoza: Acribia, 1991. 617p.

STATISTICAL Analysis System. SAS/STAT software: changes and enhancements through release 6.12. Cary: SAS Institute, 1992.

TSENKOVA, R.; ATANASSOVA, S.; ITOH, K. et al. Near infrared spectroscopy for biomonitoring cow milk composition measurement in a spectral region from 1,100 to 2,400 nanometers. J. Anim. Sci., v.78, p.515$522,2000$.

ZAR, J.H. Biostatistical analysis. 2.ed. Englewood Cliffs, NJ: Prentice Hall International; 1984. 718p. 